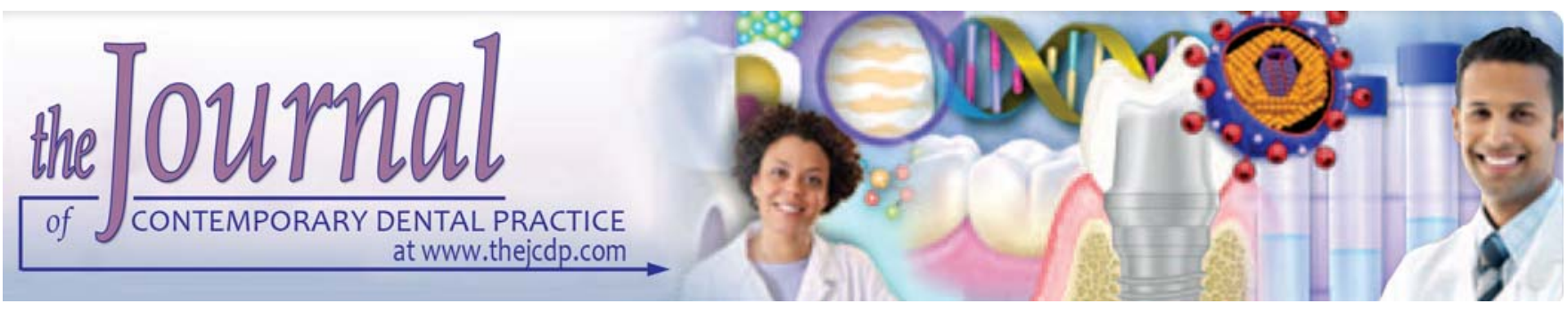

\title{
Effect of Energy Drinks on the Color Stability of Nanofilled Composite Resin
}

\author{
Ayman Al-Dharrab
}

\begin{abstract}
Objective: To study the effect of three energy drinks on the color stability of nanofilled composite resins after different periods of aging time.
\end{abstract}

Materials and methods: Composite resin disks, $6 \mathrm{~mm}$ diameter, $3 \mathrm{~mm}$ thickness were prepared from Filtec Z350 XT, Tetric EvoCeram, and Filtec Z250 XT. Specimens from each material were tested after aging with Red Bull, Bison and Power Horse energy drinks and distilled water as a control. Specimens were stored at $37^{\circ} \mathrm{C}$ in dark containers for $1,7,30$ and 60 days. Color was assessed in the CIELAB using a colorimeter before and after each storage period. Color differences $\Delta \mathrm{E}^{*}{ }_{\mathrm{ab}}$ and $\Delta \mathrm{b}^{\star}$ among specimens were measured. Mean values were statistically analyzed using one-way analysis of variance (ANOVA), with $p<0.05$ as significance level.

Results: The color change $\Delta \mathrm{E}^{\star}{ }_{\mathrm{ab}}$ and $\Delta \mathrm{b}^{\star}$ occurred by Red Bull, Bison and Power Horse energy drinks was significantly different for all tested materials at all four times $p<0.05$ except in the Red Bull group. All specimens showed increase in color changes toward yellowness $\Delta \mathrm{b}^{*}$ after a period of $1,7,30$ and 60 days. The highest total color difference $\Delta \mathrm{E}^{*}{ }_{\mathrm{ab}}$ was found in the Red Bull group after 60 days.

Conclusion: Energy drinks used in this study had staining effect on the tested composite resin materials. The discoloration increased with aging time toward yellowness; however, it was not perceptible in all test groups after 60 days.

Keywords: Energy drinks, Aging, CIELAB, Colorimeter, Nanofilled composite resins.

How to cite this article: Al-Dharrab A. Effect of Energy Drinks on the Color Stability of Nanofilled Composite Resin. J Contemp Dent Pract 2013;14(4):704-711.

Source of support: Nil

Conflict of interest: None declared

\section{INTRODUCTION}

Composite resin is being used widely as an esthetic restorative material in anterior as well as posterior teeth. ${ }^{1,2}$ Composite resin should retain the color and polish over a long period of time to serve as a long-term esthetic restorative material. Discoloration of this composite filled restoration may occurs due to intrinsic and extrinsic factors. ${ }^{3}$ Though, the quality of composite resin restorations has improved with the advent of new technology in material sciences in recent years, discoloration of the composite resin materials remains to be a major problem in long-term clinical studies. ${ }^{4-7}$ Color stability has therefore been considered as one of the most important factors when selecting composite resin materials for esthetic restorations. ${ }^{8}$

Recent studies showed that the effects of intrinsic discoloration were small in fully polymerized composite resin materials with no perceptible color changes observed after water storage alone. ${ }^{9,10}$ Significant color changes occur only when the materials are exposed to dietary colorant and chemical dyes, ${ }^{11}$ and when the composite resins are not fully polymerized. ${ }^{12,13}$ These findings suggest that extrinsic discoloration is the most important factor affecting the color stability and long-term success of composite resin restorations, which highlights the needs for dental researchers and material scientists to improve the resistance to discoloration of new resin based materials for esthetic restorations. ${ }^{8}$

Composite discoloration is a result of interaction between external colorants and the composite resin materials. The adsorption of external colorants onto the surface and the absorption into the resin matrices can both cause color changes and compromise the esthetic outcomes. ${ }^{8,14}$ The roughening of the surface caused by wear and chemical degradation may also affect gloss and consequently increase the extrinsic staining. ${ }^{15}$

Previous studies ${ }^{16,17}$ concerning color stability have shown that beverages and mouthrinses have varied degrees of staining effect on auto and light cured composite resin. The staining potential of these drinks and solutions vary according to their composition and properties. ${ }^{18}$ Over the past 10 years, the consumption of caffeinated beverages intended to energize, has increased significantly. Energy 
drinks were recently shown to comprise $20 \%$ of the total convenience store beverage market, with 'Red Bull' and ' $\mathrm{V}$ ' accounting for over $97 \%$ of sales in this multimilliondollar industry. The main active constituents of energy drinks include varying amount of caffeine, guarana extract, taurine and ginseng. Additional amino acids, vitamins and carbohydrate usually complete the list of purportedly beneficial ingredients. ${ }^{19}$

To determine and quantify the changes in the color of dental materials, an understanding of color space and differential colorimetry is required. Current photometric and colorimetric instruments are capable of reliably quantifying the color of acrylic resin specimens. ${ }^{20}$ Photometric and colorimetric instruments measure color and express it in terms of three coordinate values $\left(\mathrm{L}^{*}, \mathrm{a}^{*}, \mathrm{~b}^{*}\right)$, which locate the object's color within the CIELAB color space. The $\mathrm{L}^{*}$ coordinate represents the brightness of an object, the $\mathrm{a}^{*}$ value represent the red or green chroma, and the $b^{*}$ value represents the yellow or blue chroma. The color difference $(\Delta \mathrm{E})$ of two objects can then determine by comparing the differences between respective coordinate values for each object. The formula used for calculating color differences in this system is: $:^{20,21}$

$$
\Delta \mathrm{E}^{*}{ }_{\mathrm{ab}}=\left[\left(\Delta \mathrm{L}^{*}\right)^{2}+\left(\Delta \mathrm{a}^{*}\right)^{2}+\left(\Delta \mathrm{b}^{*}\right)^{2}\right]^{1 / 2}
$$

Where $\mathrm{L}^{*}, \mathrm{a}^{*}$ and $\mathrm{b}^{*}$ are differences in color parameters for the two specimens measured for comparison. Numeric description of color permits precise definition of the magnitude of the color difference between objects.

Previous studies reported different acceptability and perceptibility thresholds for color differences in dental materials. ${ }^{21}$ The magnitude of the color difference is based on the human perception of color. It was previously reported that color differences greater than $1 \Delta \mathrm{E}$ unit are visually perceivable by $50 \%$ of human observers. ${ }^{20}$ However, under uncontrolled clinical conditions, such small differences in color would be unperceivable, as average color differences below 3.7 are rated a 'match' in clinical conditions. ${ }^{22}$
Therefore, the aim of this study was to evaluate the color stability of nanofilled composite resins with the effect of three energy drinks after different periods of aging time.

\section{MATERIALS AND METHODS}

In present study, three types of nanofilled composite resins that are used for anterior and posterior esthetic restorations were selected. This included Filtec Z350 XT, Tetric EvoCeram, and Filtec Z250 XT (Table 1). A total of 80 disks shaped specimens, $6 \mathrm{~mm}$ in diameter and $3 \mathrm{~mm}$ thick were prepared for each composite resin material using a customized cylindrical Teflon mold. After packing the composite material into the mold with an incremental technique, a polyester strip was pressed onto the mold surface with a glass plate to obtain a flat surface without bubble formation. Each composite material specimen was polymerized from both sides for 20 seconds using a LED light curing unit (3M ESPE Dental Products D-82229 Seefeld, Germany) operating in standard mode and emitting not less than $600 \mathrm{~mW} / \mathrm{cm}^{2}$ as directed by the manufacturer. Emitting light was measured with a light meter that was placed on the curing unit before beginning of polymerization. The guide of the light curing unit was placed perpendicular to the composite resins specimen surface and the distance between the light source and specimen was standardized using a $1 \mathrm{~mm}$ glass slide. All the specimens were then polished using soflix disks (3M ESPE Sof-Lex, Germany) as follow: the top surfaces were sequentially polished with medium, fine and superfine aluminum oxide impregnated disks using a slow speed headpiece under dry conditions for 30 seconds. After each polishing step, the specimens were thoroughly rinsed with water for 10 seconds to remove debris, air dried for 5 seconds, and then polished with another disk of lower grit for the same period of time. All prepared specimens were then stored in distilled water for 24 hours at $37^{\circ} \mathrm{C}$ to ensure complete polymerization.

Table 1: Details of composite resin materials and energy drinks used in this study

\begin{tabular}{|c|c|c|c|}
\hline Materials & Type & Shade & Manufacturer \\
\hline Filtek Z350 XT & Universal nanocomposite & $A_{2}$ & 3M ESPE Dental Products D-82229 Seefeld -Germany. \\
\hline Tetric EvoCeram & Universal nanohybrid composite & $A_{2}$ & Ivoclar Vivadent AG, FL-9494 Schaan \\
\hline Filtek Z250 XT & Nanohybrid composite & $A_{2}$ & 3M ESPE Dental Products D-82229 Seefeld -Germany. \\
\hline Red Bull & $\begin{array}{l}\text { Energy drink (Sucrose, glucose, acidity } \\
\text { regulatory sodium, caffeine, vitamins, } \\
\text { natural flavors, colors) }\end{array}$ & - & Red Bull GmbH, Austria \\
\hline Bison & $\begin{array}{l}\text { Energy drink (carbonated water, sugar, } \\
\text { citric acid, caffeine, sodium benzoate, } \\
\text { vitamins, natural flavors, colors) }\end{array}$ & - & Abuljadayal Beverages INC, Jeddah, Saudi Arabia \\
\hline P Horse & $\begin{array}{l}\text { Energy drink (Carbonated water, } \\
\text { sucrose, glucose, citric acid, taurine, } \\
\text { caffeine, color, inositol, niacin, pantothenic } \\
\text { acid, vitamins) }\end{array}$ & - & S.Spitz GmbH, Attnang-Puchheim, Austria \\
\hline
\end{tabular}


Specimens from each composite material were subdivided into four groups of 20. Specimens from each group were immersed in vials containing $30 \mathrm{ml}$ of the following energy drinks; Red Bull, Bison, Power Horse, and distilled water (control) for 1, 7, 30 and 60 days at $37^{\circ} \mathrm{C}$ temperature. At the end of each storage period, the specimens were dipped 10 times in cleansing solution, consisting of $10 \mathrm{ml}$ soap and $700 \mathrm{ml}$ distilled water and subsequently flushed with running tap water and then the excess water on the surfaces was removed by gentle drying by tissue paper. The specimens were then allowed to stand for 1 hour at ambient room temperature. The aging solutions were renewed daily and $\mathrm{pH}$ value was monitored before immersing the specimens.

Color measurements of all specimens were performed according to the CIE $\mathrm{L}^{*} \mathrm{a}{ }^{*} \mathrm{~b}$ color scale relative to the standard illuminant D65 over a white background with a colorimeter (Konica Minolta CR-400/410; Minolta Co, Osaka, Japan) using CIE (Commission International de1 Eclairage). L*a *b* relative to standard, illuminate A, against a white background. Since color differences were being tested, the choice of the illuminate was not important.

Before each tested material measurements, the colorimeter was calibrated according to the manufacturer's recommendations by using the supplied white calibration standard. The spectrophotometer was set to automatically calculate the mean color of the specimens of each material. The CIE L* a* b* color system is a three-dimensional color measurement system, where $\mathrm{L}^{*}$ refers to the lightness coordinate, which has a value ranging from 0 for perfect black to 100 for perfect white and $\mathrm{a}^{*}$ and $\mathrm{b}^{*}$ are the chromaticity coordinates on the green-red $\left(-a^{*}=\right.$ green, $+a^{*}$ $=$ red $)$ and blue-yellow $\left(-b^{*}=\right.$ blue; $+b^{*}=$ yellow $)$ axes respectively. ${ }^{1,20}$

All color measurements were obtained by one operator. The color measurements were performed at the baseline and at a time interval of 1 day, 7, 30 and 60 days periods. All the specimens were placed in the viewing port of the spectrophotometer. The $\mathrm{L}^{*}, \mathrm{a}^{*}$, and $\mathrm{b}^{*}$ values of each specimen after immersion for the specific time period were measured five times by placing each specimen on the measuring head and covering it with the black cover.

The mean value of $\Delta \mathrm{L}^{*}, \Delta \mathrm{a}^{*}$ and $\Delta \mathrm{b}^{*}$ after five measurements were automatically calculated by the spectrophotometer and recorded. The color difference $\Delta \mathrm{E}$ was calculated from the mean $\Delta \mathrm{L}^{*}, \Delta \mathrm{a}^{*}$ and $\Delta \mathrm{b}^{*}$ values for each specimen using the following formula: ${ }^{20}$

$$
\Delta \mathrm{E}^{*}\left(\mathrm{~L}^{*} \mathrm{a} * \mathrm{~b} *\right)=\left[(\Delta \mathrm{L} *)^{2}+\left(\Delta \mathrm{a}^{*}\right)^{2}+(\Delta \mathrm{b} *)^{2}\right]^{1 / 2}
$$

Where $\Delta \mathrm{L}^{*}, \Delta \mathrm{a}^{*}$ and $\Delta \mathrm{b}^{*}$ are the differences in the $\mathrm{L}^{*} \mathrm{a}^{*}$ and $b^{*}$ values, respectively before and after immersion for each time period.

The mean data obtained from the specimens of the materials aged with the test groups at different interval times were statistically analyzed. Mean values of the different groups at different aging times were compared using oneway analysis of variance (ANOVA) and multiple comparisons of the mean values were performed using the Tukey test and $\mathrm{p}<0.05$ was considered as the level of significance.

\section{RESULTS}

The mean of the color change values $\left(\Delta \mathrm{E}^{*}{ }_{\mathrm{ab}}\right)$ for the tested resin composite materials following the immersion in the different solutions for 1, 7, 30 and 60 days are summarized in Tables 2 to 5 and graphically presented in Graphs 1 to 4 . Among all the tested composite materials, it was consistently observed that Tetric EvoCeram specimens which were immersed in Power Horse for 1 day revealed the lowest $\Delta \mathrm{E}^{*}{ }_{\mathrm{ab}}$ values, whereas the highest $\Delta \mathrm{E}^{*}{ }_{\mathrm{ab}}$ values were observed in the Z250 XT specimens after being immersed in Red Bull for 60 days. When comparing the three composite materials immersed in Red Bull, no significant differences were observed after immersion for periods of $1,7,30$ and 60 days $(p=0.133)$. Significant differences were only observed for the materials Z350 XT, Tetric EvoCeram and Z250 XT after the period of 1, 7, 30 and 60 days in the specimens immersed in Bison and Power

\begin{tabular}{|c|c|c|c|c|c|}
\hline Materials & $\begin{array}{l}\text { Total color } \\
\text { diff. in } 1 \text { day }\end{array}$ & $\begin{array}{c}\text { Total color } \\
\text { diff. in } 7 \text { days }\end{array}$ & $\begin{array}{c}\text { Total color } \\
\text { diff. in } 30 \text { days }\end{array}$ & $\begin{array}{c}\text { Total color } \\
\text { diff. in } 60 \text { days }\end{array}$ & Significant level \\
\hline \multicolumn{6}{|c|}{$\Delta E^{\star} a b$} \\
\hline $\mathrm{Z} 350 \mathrm{XT}$ & 0.51 & 0.58 & 1.06 & 1.07 & $p=0.133$ \\
\hline Tetric EvoCeram & 0.40 & 0.39 & 0.92 & 0.92 & \\
\hline $\mathrm{Z} 250 \mathrm{XT}$ & 0.61 & 0.57 & 1.08 & 1.27 & \\
\hline \multicolumn{6}{|c|}{$\Delta b^{\star}$} \\
\hline $\mathrm{Z} 350 \mathrm{XT}$ & 0.45 & 0.37 & 0.31 & 1.02 & $p<0.001$ \\
\hline Tetric EvoCeram & 0.20 & 0.22 & 0.21 & 0.88 & \\
\hline $\mathrm{Z} 250 \times \mathrm{T}$ & 0.39 & 0.42 & 0.38 & 1.22 & \\
\hline
\end{tabular}




\begin{tabular}{|c|c|c|c|c|c|}
\hline Materials & $\begin{array}{c}\text { Total color } \\
\text { diff. in } 1 \text { day }\end{array}$ & $\begin{array}{c}\text { Total color } \\
\text { diff. in } 7 \text { days }\end{array}$ & $\begin{array}{c}\text { Total color } \\
\text { diff. in } 30 \text { days }\end{array}$ & $\begin{array}{c}\text { Total color } \\
\text { diff. in } 60 \text { days }\end{array}$ & Significant level \\
\hline \multicolumn{6}{|c|}{$\Delta E^{\star} a b$} \\
\hline $\mathrm{Z} 350 \mathrm{XT}$ & 0.20 & 0.65 & 1.04 & 1.00 & $p<0.001$ \\
\hline Tetric EvoCeram & 0.18 & 0.54 & 0.86 & 0.90 & \\
\hline $\mathrm{Z} 250 \mathrm{XT}$ & 0.22 & 0.62 & 0.95 & 0.99 & \\
\hline \multicolumn{6}{|c|}{$\Delta b^{*}$} \\
\hline Z350 XT & -0.03 & 0.10 & 0.18 & 0.87 & $p<0.001$ \\
\hline Tetric EvoCeram & 0.03 & 0.04 & 0.12 & 0.74 & \\
\hline $\mathrm{Z} 250 \mathrm{XT}$ & 0.11 & 0.10 & 0.18 & 0.88 & \\
\hline
\end{tabular}

\begin{tabular}{|c|c|c|c|c|c|}
\hline Materials & $\begin{array}{c}\text { Total color } \\
\text { diff. in } 1 \text { day }\end{array}$ & $\begin{array}{c}\text { Total color } \\
\text { diff. in } 7 \text { days }\end{array}$ & $\begin{array}{c}\text { Total color } \\
\text { diff. in } 30 \text { days }\end{array}$ & $\begin{array}{c}\text { Total color } \\
\text { diff. in } 60 \text { days }\end{array}$ & Significant level \\
\hline \multicolumn{6}{|c|}{$\Delta E^{\star} a b$} \\
\hline Z350 XT & 0.39 & 0.48 & 0.57 & 1.04 & $P=0.003$ \\
\hline Tetric EvoCeram & 0.14 & 0.34 & 0.51 & 0.65 & \\
\hline $\mathrm{Z} 250 \mathrm{XT}$ & 0.26 & 0.49 & 0.71 & 0.97 & \\
\hline \multicolumn{6}{|c|}{$\Delta b^{*}$} \\
\hline Z350 XT & 0.34 & 0.40 & 0.40 & 0.85 & $P=0.009$ \\
\hline Tetric EvoCeram & 0.05 & 0.12 & 0.08 & 0.57 & \\
\hline $\mathrm{Z} 250 \times \mathrm{X}$ & 0.25 & 0.32 & 0.34 & 0.92 & \\
\hline
\end{tabular}

\begin{tabular}{|c|c|c|c|c|c|}
\hline Materials & $\begin{array}{c}\text { Total color } \\
\text { diff. in } 1 \text { day }\end{array}$ & $\begin{array}{c}\text { Total color } \\
\text { diff. in } 7 \text { days }\end{array}$ & $\begin{array}{c}\text { Total color } \\
\text { diff. in } 30 \text { days }\end{array}$ & $\begin{array}{c}\text { Total color } \\
\text { diff. in } 60 \text { days }\end{array}$ & Significant level \\
\hline \multicolumn{6}{|c|}{$\Delta E^{\star} a b$} \\
\hline $\mathrm{Z} 350 \mathrm{XT}$ & 0.14 & 0.76 & 0.77 & 0.63 & $p<0.001$ \\
\hline Tetric EvoCeram & 0.14 & 0.81 & 0.92 & 0.80 & \\
\hline $\mathrm{Z} 250 \mathrm{XT}$ & 0.15 & 0.84 & 0.98 & 0.82 & \\
\hline \multicolumn{6}{|c|}{$\Delta b^{*}$} \\
\hline $\mathrm{Z} 350 \mathrm{XT}$ & -0.08 & -0.16 & -0.06 & 0.39 & $p<0.001$ \\
\hline Tetric EvoCeram & 0.04 & -0.04 & 0.04 & 0.47 & \\
\hline $\mathrm{Z} 250 \mathrm{XT}$ & -0.03 & -0.14 & -0.06 & 0.38 & \\
\hline
\end{tabular}

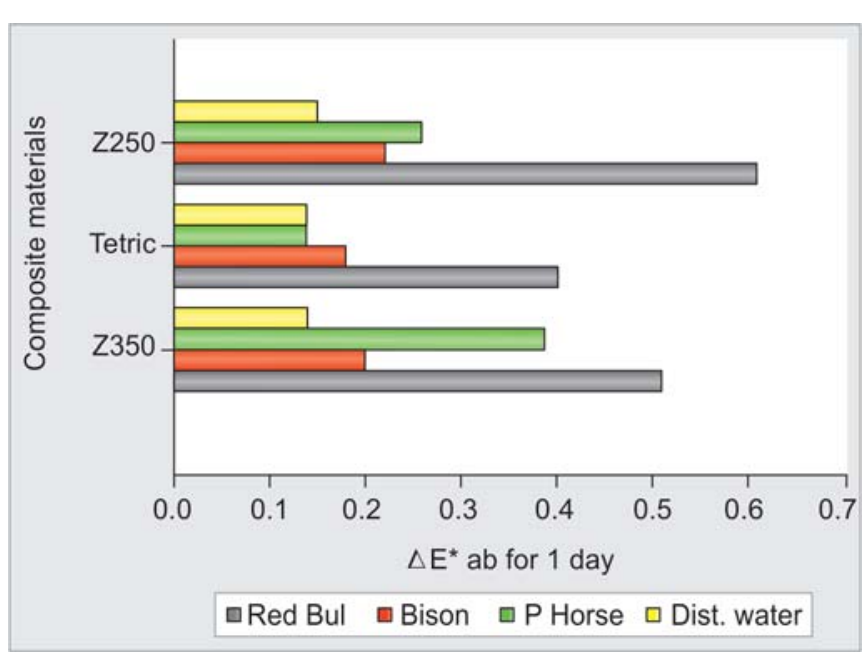

Graph 1: Total color difference $\Delta \mathrm{E}^{*}$ ab for the tested resin composites exposed to different energy drinks for a period of 1 day

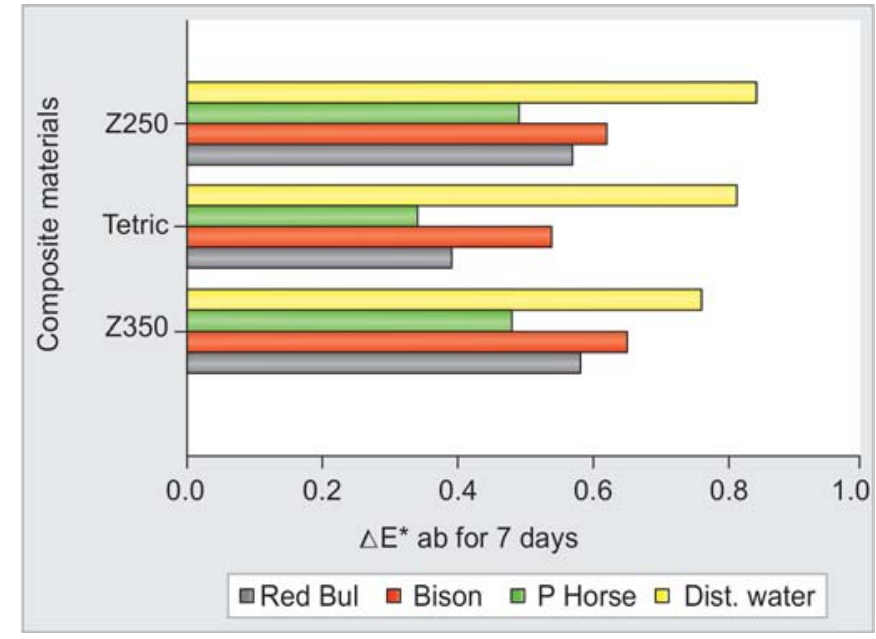

Graph 2: Total color difference $\Delta \mathrm{E}^{\star}$ ab for the tested resin composites exposed to different energy drinks for a period of 7 days 


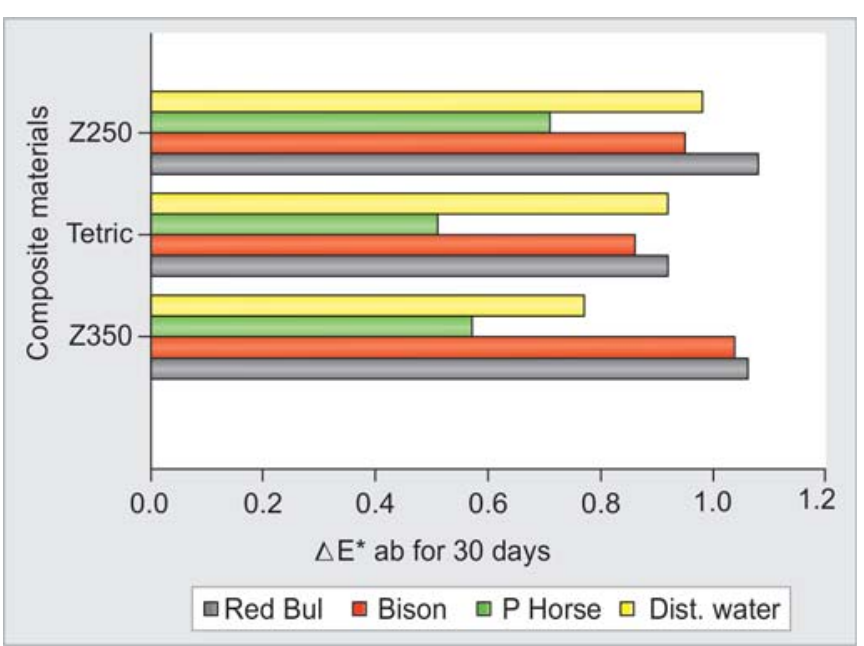

Graph 3: Total color difference $\Delta E^{\star}$ ab for the tested resin composites exposed to different energy drinks for a period of 30 days

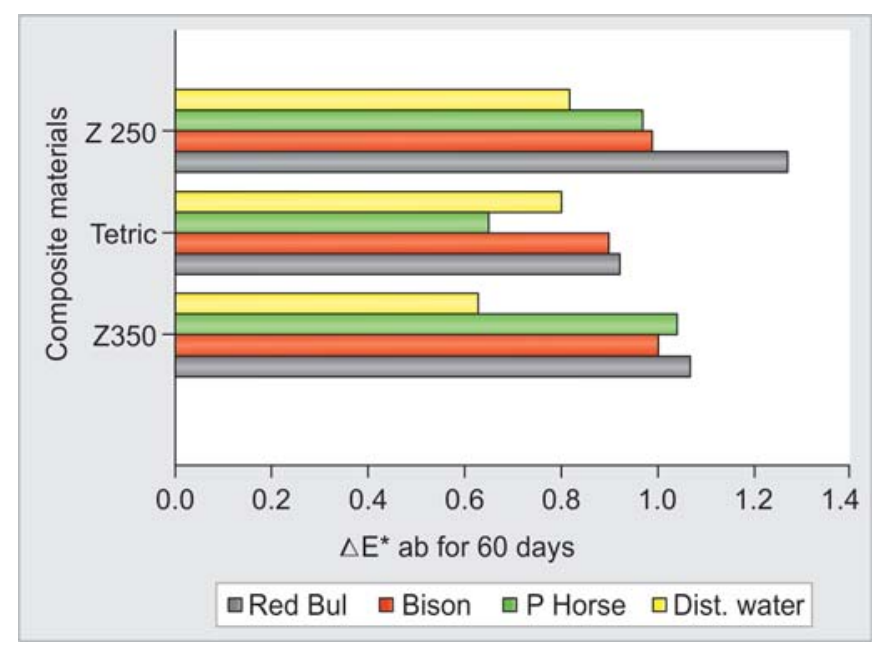

Graph 4: Total color difference $\Delta \mathrm{E}^{\star}$ ab for the tested resin composites exposed to different energy drinks for a period of 60 days

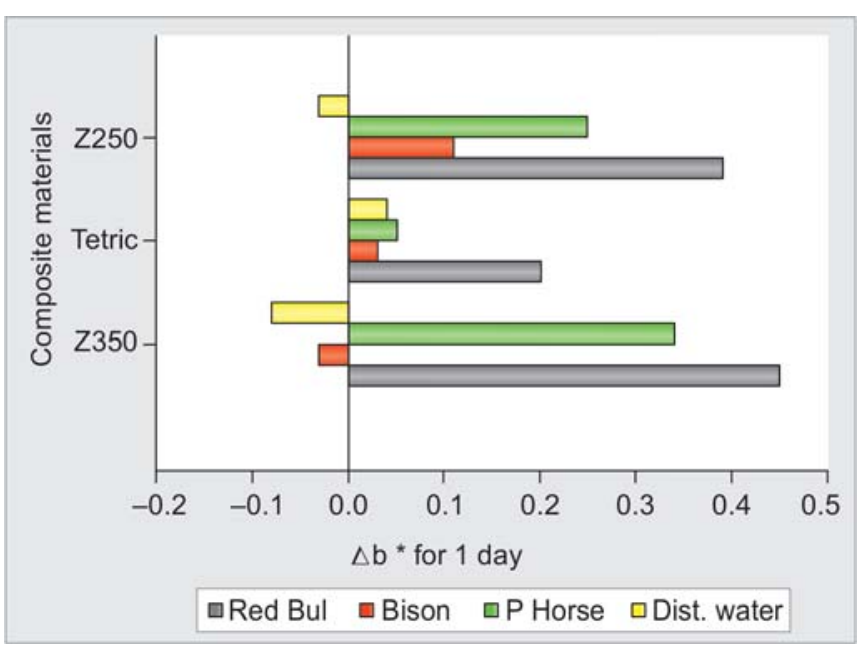

Graph 5: Color difference $\Delta b^{\star}$ of the resin composites aged for 1 day with different energy drinks

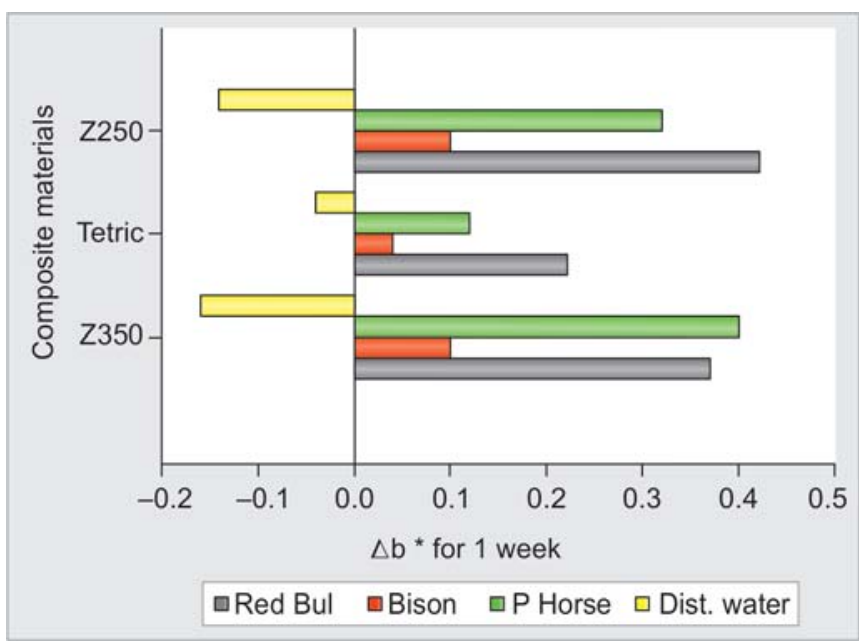

Graph 6: Color difference $\Delta b^{*}$ of the resin composites aged for 7 days with different energy drinks

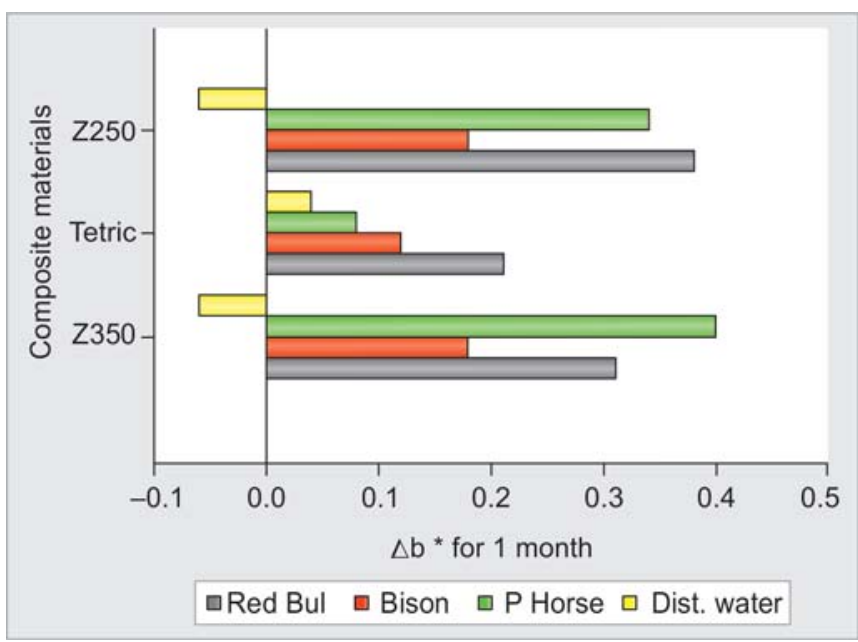

Graph 7: Color difference $\Delta b^{*}$ of the resin composites aged for 30 days with different energy drinks

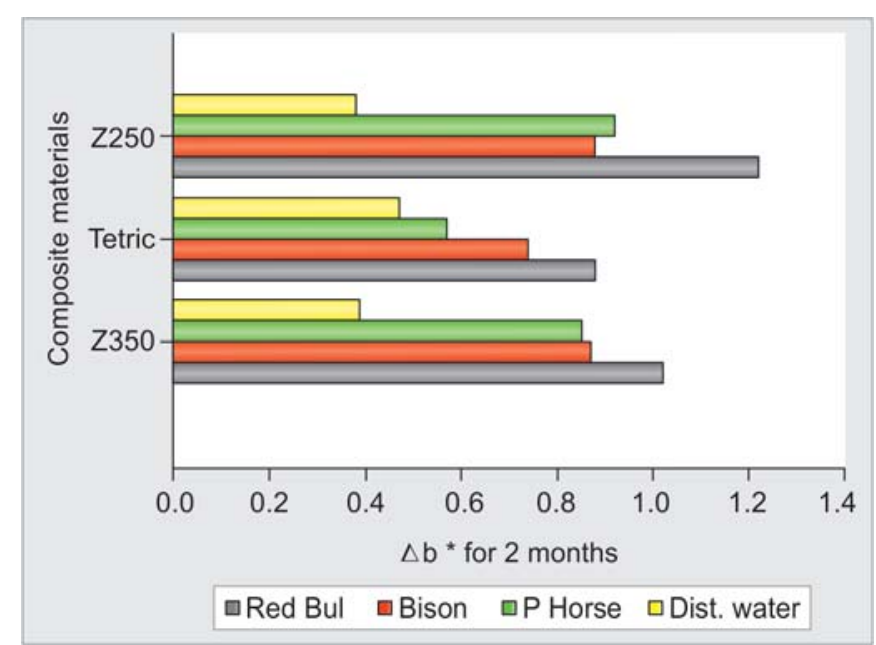

Graph 8: Color difference $\Delta b^{\star}$ of the resin composites aged for 60 days with different energy drinks 
Horse sports drinks, $\mathrm{p}<0.001$ and $\mathrm{p}=0.003$ respectively (Tables 3 and 4). For specimens immersed in distilled water, significant color difference was observed between the materials after 60 days ( $p<0.001)$. All tested materials revealed an increase in the total color difference $\Delta \mathrm{E}_{\mathrm{ab}}$ with time, however, this was clinically acceptable $\left(\Delta \mathrm{E}_{\mathrm{ab}}<3.3\right)$ after 60 days of aging.

In respect to $\Delta \mathrm{b}^{*}$ (Change along yellow-blue axis) values where positive $\Delta \mathrm{b}^{*}$ indicates a shifts toward yellow color whilst negative $\Delta b^{*}$ denotes a shift toward blue color. Tables 2 to 5 and Figures 5 to 8 represent the mean color measurement differences $\Delta \mathrm{b} *$ for all tested resin composite materials in different test conditions after 1, 7, 30 and 60 days. A significant change was noted for all the materials after all periods of aging ( $\mathrm{p}<0.05)$.

After 1 day of immersion, all tested materials showed positive $\Delta b^{*}$ discoloration toward yellow axis except for Z350 XT that was stored in Bison showed a negative $\Delta \mathrm{b}^{*}$. After 1 day of immersion, Z350 XT that was stored in Red Bull showed the highest discoloration as can be shown in Graph 5.

After 7 days of immersion in energy drinks, all materials showed an increase shift toward yellow axis ( $\Delta \mathrm{b}^{*}$ positive), the Z350 XT and Z250 XT materials being the heist material discolored, while materials immersed in distilled water revealed a discoloration towards blue axis (low values) as can be shown in Graph 6.

After 30 days of immersion, a maximum change in $\Delta b^{*}$ for all materials was seen in Z350 XT composite resin material that immersed in Power Horse solution ( $\mathrm{p}<0.009)$. All materials immersed in Red Bull and power horse solutions after $1,7,30$ and 60 days $\Delta b^{*}$ showed an increased toward yellow axis (positive $\Delta b^{*}$ ) as presented in Graph 7 .

Graph 8 illustrates the response of the tested materials after immersion in energy drinks after 60 days. All tested composite resin materials immersed in aging solutions revealed increase of $\Delta \mathrm{b}^{*}$ toward yellow axis. $\mathrm{Z} 250 \mathrm{XT}$ that immersed in Red Bull ( $\mathrm{p}<0.001)$, Bison ( $<<0.001)$, and power horse $(\mathrm{p}<0.009)$ exhibited the highest $\Delta \mathrm{b}^{*}$ then Z350 XT and followed by Tetric $\left(\Delta \mathrm{b}^{*} \geq 1.5\right)$.

\section{DISCUSSION}

Color has an important role in obtaining optimum esthetics. ${ }^{23}$ An increase in the demand from patients for improved esthetics has resulted in the development of restorative materials with excellent esthetic properties and their widespread use in dental practice. However, a major disadvantage of resin composites is their tendency to discolor, which may be a major factor in the replacement of restorations. ${ }^{24,25}$ Therefore, restorative materials should match well the initial shade and preserve the esthetic semblance overtime in the restored tooth. ${ }^{23}$ In the present study, the color stability of the three nanofilled resin composites, all of which can be used for anterior and posterior applications in dental practices, was assessed under the actions of acidic energy drinks, which are commonly consumed by the general population. The three resin tested composite materials in this study revealed significant color changes after 60 days of immersion in the three types of solutions.

The color of the Tetric EvoCeram resin composite was the least affected by the immersion, whereas the Filtec Z250 XT resin composite was the most prone to color change. The discolorations of the composite materials were related to the resin filler type, type of resin matrix and type of staining agent. ${ }^{26}$ Resin composite materials that can absorb water are also capable of absorbing other fluids with pigments, resulting in discoloration. It is assumed that water acts as a conductor for the pigment and stain penetration into the resin matrix. ${ }^{27,28}$ Although, the resin matrix of the composite materials can absorb water from the environment into the bulk of their structure, inorganic glass fillers cannot absorb water into the bulk of the material, but just absorb water on their surface. Excessive water sorption may decrease the life of a resin composite by expanding and plasticizing the resin component, hydrolyzing the silane, and causing micro-crack formation. As a result, the microcracks or interfacial gaps at the interface between the filler and matrix allow stain penetration and discoloration. ${ }^{27}$

Owing to the acidity and erosive potential of energy drinks, ${ }^{29}$ the composite specimens were immersed in the sports drinks for specified periods of 1, 7, 30 and 60 days to evaluate the color change that can be assessed both visually and by specific instruments. The surface quality and susceptibility to external discoloration in this experiment was investigated. The same procedure and measurements were carried out on each type of material at the three conditions (aging beverages), using the same equipment. The methodology used in the present study was in accordance with previous studies that used calorimeter (Menolta) and the CIE L*a*b* coordinate system, which is a recommended method for dental purposes. ${ }^{23}$ The CIE $\mathrm{L} * \mathrm{a} * \mathrm{~b} *$ coordinate system was chosen to evaluate the color variation $\left(\Delta \mathrm{E}^{*}{ }_{\mathrm{ab}}\right)$ because it is well-suited for the determination of small color changes and has advantages such as repeatability, sensitivity and objectivity. ${ }^{30}$

The study of $\Delta \mathrm{E}^{*}$ values (Tables 2 to 5 ) showed that all of the tested materials had values of $\Delta \mathrm{E}^{*}{ }_{\mathrm{ab}}<3.3$. The human eye cannot detect (visually perceptible) $\Delta \mathrm{E}$ values less than 3.3 and this value is measurable with the help of colorimeter if it is less than this value. ${ }^{30}$ 
The color shift in different test groups was significantly different in composites immersed with Bison ( $p<0.001)$ and P Horse $(\mathrm{p}=0.003)$ except materials immersed with Red Bull ( $p=0.133)$.

When the color change of the tested materials that immersed in Red Bull in a period of 1, 7, 30 and 60 days was not perceivable by the human eye $\Delta \mathrm{E}^{*}{ }_{\mathrm{ab}}<1.5$, where the highest was Z250 $\Delta \mathrm{E}^{*}{ }_{\mathrm{ab}} 1.27$ (clinically acceptable), and statistically not significant values ( $p=0.133$ ).

$\Delta \mathrm{E}^{*}{ }_{\mathrm{ab}}$ in specimens immersed in Bison for the assigned periods were not perceivable by the human eye in the all periods of aging $\Delta \mathrm{E}^{*}{ }_{\mathrm{ab}}<1.5$, for the all composite materials $\mathrm{Z} 350 \mathrm{XT}$ revealed $\Delta \mathrm{E}^{*}{ }_{\mathrm{ab}} 1.00, \mathrm{Z} 250 \mathrm{XT} \Delta \mathrm{E}^{*}{ }_{\mathrm{ab}} 0.99$, and Tetric EvoCeram $\Delta \mathrm{E}^{*}{ }_{\mathrm{ab}} 0.90$ which is not perceivable by the human eye in the period of 60 days of aging $\Delta \mathrm{E}^{*}{ }_{\mathrm{ab}}$ $<3.3$, where all the materials increases in the $\Delta \mathrm{E}^{*}{ }_{\mathrm{ab}}$ and statistically highly significant $(\mathrm{p}<0.001)$ as tabulated in Table 3 and graphically in Graphs 1 to 4 .

The total color difference $\Delta \mathrm{E}^{*}{ }_{\mathrm{ab}}$ in specimens immersed in Power Horse beverages was also increasing by time and statistically significant ( $p=0.003$ ). The discoloration was clinically acceptable and not perceivable by the human eye in the all periods of aging where $\Delta \mathrm{E}^{*}{ }_{\mathrm{ab}}<3.3$ (Table 4 , Graphs 1 to 4$)$.

According to the previous studies, It was reported that minimum differences in the CIE LAB color parameters $\left(\Delta \mathrm{L}^{*}, \Delta \mathrm{a}^{*}\right.$ and $\Delta \mathrm{b}^{*}$ ) of $1 \mathrm{CIE}$ unit would be perceptible to an observer depending on the CIE $\mathrm{L}^{*}$ value, background color and lighting, while differences in the range 1 to $2 \mathrm{CIE}$ units would be noticeable to most observers. ${ }^{8}$

Color changes values of $\Delta \mathrm{b}^{*}$ in the tested composite resin materials immersed in Red Bull for a period of 1,7 , 30 and 60 days was not perceivable by the human eye in the all periods $\Delta b^{*}<1.5$, where all the materials showed increased discoloration toward yellowness (positive values) axis with the increase of aging time. The highest value was Z250 $\Delta \mathrm{b}^{*} 1.22$ (clinically acceptable), followed by Z350 XT $\Delta \mathrm{b} * 1.02$, and Tetric EvoCeram $\Delta \mathrm{b} * 0.88$. It shows statistically highly significant values ( $<<0.001)$. The effect of the Red Bull was high on all materials were the color shifts toward the yellowness axis same as the other types of energy drinks where it discolored toward the yellow axis increasing with the aging time, due to the presence of acidic in the component of the energy drinks (Table 2, Graphs 5 to 8).

In respect to the composite materials immersed in Bison energy drink for the assigned periods, the color shifting of $\Delta \mathrm{b}^{*}$ for the materials was highly statistically significant $(\mathrm{p}<0.001)$ it was increasing toward yellow axis (positive value), not perceivable by the human eye in the all periods of aging periods where $\Delta \mathrm{b}^{*}<1.5$ and this is clinically acceptable (Table 3, Graphs 5 to 8).

While the $\Delta b^{*}$ for the composite materials aged in Power Horse energy drinks in a period, the discoloration was lower than other groups and minimal and increasing toward the yellowness, clinically acceptable, and statistically significant $(\mathrm{p}=0.009)$.

Since it has been widely accepted in industry, the CIELAB system provides dentistry with a standardized method for predicting the visual response to color differences, and for describing the shifts in color space responsible for those differences.

The present study is in agreement with previous findings ${ }^{11,23} \Delta \mathrm{E}^{*}{ }_{\mathrm{ab}}$ values equal to or greater than 3.3 were considered clinically perceptible, as previously reported. Our study demonstrated that the tested solutions, induced varying degrees of discoloration in the resin composites tested after periods of 1, 7, 30 and 60 days.

The results of this study as well as of others support the reliability of measurement of colorimetric technology using the CIELAB system.

\section{CONCLUSION}

Within the limitation of this in vitro study, it can be concluded that all energy drink solutions used in this study affected the color stability of tested resin composites materials with the increasing of aging time. The effect of energy drinks solutions on the color stability of resin composites depends on type of solution and the presence of the acids in the composition with the aging time. Further studies with longer period of aging are warranted by the findings of this study.

\section{REFERENCES}

1. Rajkumar K, Kumar S, Mahalaxmi S, Ragavi P, Mageshwaran TA. Colour stability of resin composites after emersing in coffee of different temperature-an in vitrostudy. SRM University Journal of Dental Sciences 2011;2:91-95.

2. Ertan ERTA, et al. Colour stability of resin composites after immersion in different drinks. Dental Materials Journal 2006; 25:371-376.

3. Bencourt $\mathrm{S}$, et al. Characterization of water sorption, solubility and filler particles of light cured composite resins. Braz Dent J 2009;20(4):314-318.

4. Van Dijken JW. Durability of new restorative materials in Class III cavities. Journal of Adhesive Dentistry 2001;3:65-70.

5. Millar BJ, Robinson PB, Inglis AT. Clinical evaluation of an anterior hybrid composite resin over 8 years. Br Dent J 1997;182:26-30.

6. Lucarotti PSK, Holder RL, Burke FJT. Outcome of direct restorations placed within the general dental services in England and Wales (Part 1): variation by type of restoration and reintervention. Journal of Dentistry 2005;33:805-815. 
7. Demirci M, Sancakli HS, Uysal O. Clinical evaluation of a polyacid-modified resin composite (Dyract) in class $\mathrm{V}$ carious lesions: 5-year results. Clinical Oral Investigations 2008;12:157163.

8. Ren YF, Feng L, Serban D, Malmstrom HS. Effects of common beverage colorants on color stability of dental composite resins: The utility of a thermocycling stain challenge model in vitro. Journal of Dentistry 2012;40:e46-e48.

9. Bagheri R, Burrow MF, Tyas M. Influence of food-simulating solutions and surface finish on susceptibility to staining of aesthetic restorative materials. Journal of Dentistry 2005;33: 389-398.

10. Barutcigil C, Yýldýz M. Intrinsic and extrinsic discolouration of dimethacrylate and silorane based composites. Journal of Dentistry 2012;40(Suppl. 1):e57-63.

11. Park J-K, Kim T-H, Ko C-C, Garcia-Godoy F, Kim H-I, KwonYH. Effect of staining solutions on discoloration of resin nanocomposites. Am J Dent 2010;23:39-42.

12. Celik EU, Aladag A, Turkun LS, Yilmaz G. Color changes of dental resin composites before and after polymerization and storage in water. Journal of Esthetic \& Restorative Dentistry 2011;23:179-188.

13. Gaintantzopoulou M, Kakaboura A, Vougiouklakis G. Colour stability of tooth-coloured restorative materials. European Journal of Prosthodontics \& Restorative Dentistry 2005;13:51-56.

14. Cesar Penazzo LEPRI, Regina Guenka PALMA-DIBB. Surface roughness and color change of a composite: inuence of beverages and brushing. Dent Mater J 2012;31(4):689-696.

15. Van Groeningen G, Jongebloed W, Arends J. Composite degradation in vivo. Dent Mater 1986;2:225-227.

16. Türkün LS, Türkün M. Effect of bleaching and repolishing procedures on coffee and tea stain removal from three anterior composite veneering materials. J Esthet Restor Dent 2004;16: 290-301.

17. Manabe A, Kato Y, Finger WJ, Kanehira M, Komatsu M. Discoloration of coating resins exposed to staining solutions in vitro. Dent Mater J 2009;28:338-343.

18. Guler AU, Yilmaz F, Kulunk T, Guler E, Kurt S. Effects of different drinks on stainability of resin composite provisional restorative materials. J Prosthet Dent 2005;94:118-124.
19. Gunja N, Brown JA. Energy drinks: health risks and toxicity. MJA 2012;196(1):46-49.

20. Imirzalioglu P, Karacaer O, Yilmaz B, Ozmen L. Color Stability of Denture Acrylic Resins and a soft lining material against tea, coffee and nicotine. J Prosthodont 2010;19:118-124.

21. O’Brien WJ. Dental materials and their selection, 3rd ed. Chicago, Quintessence, 2002; pp. 24-36, 210-238.

22. Johnston WM, Kao EC. Assessment of appearance match by visual observation and clinical colorimetry. J Dent Res 1989; 68:819-822.

23. Nasim I, Neelkantan P, Sujeer R, Subbarao CV. Color stability of microfilled, microhybrid and nanocomposite resins - an in vitro study. Journal of Dentistry 2010;38:e137-142.

24. Al-Negrish AR. Composite resin restorations: a cross-sectional survey of placement and replacement in Jordan. International Dental Journal 2002;52:461-468.

25. Craig RG, Sakaguchi R. Resin composite restorative materials. 12th ed. Mosby; 2006. pp 189-212.

26. Erdemir U, Yildiz E, Eren MM. Effect of sports drinks on color stability of nanofilled and microhybrid composites after longterm immersion. Journal of Dentistry 2012;40(2):e55-e63.

27. Bagheri R, Burrow MF, Tyas M. Influence of food simulating solutions and surface finish on susceptibility to staining of aesthetic restorative materials. Journal of Dentistry 2005;33:389-398.

28. Schulze KA, Marshall SJ, Gansky SA, Marshall GW. Color stability and hardness in dental composites after accelerated aging. Dental Materials 2003;19:612-619.

29. Coombes JS. Sports drinks and dental erosion. Am J Dent 2005;18:101-104.

30. Brook AH, Smith RN, Lath DJ. The clinical measurement of tooth color and stain. Int Dent J 2007;57:324-330.

\section{ABOUT THE AUTHOR}

\section{Ayman Al-Dharrab}

Assistant Professor and Consultant, Department of Oral and Maxillofacial Rehabilitation, Faculty of Dentistry, King Abdulaziz University, Jeddah-21589, Saudi Arabia, e-mail: aaldharab@kau.edu.sa 